\title{
The development of a model of pandemic preparedness planning utilizing critical success factors from the United States and the European Union
}

\author{
Y. Draine ${ }^{1}$, J. Johnson ${ }^{1}$, M. Levy ${ }^{2} \&$ W. Sumrall ${ }^{3}$ \\ ${ }^{I}$ Department of Health Administration, \\ Central Michigan University, USA \\ ${ }^{2}$ Public Health Program, University of Memphis, USA \\ ${ }^{3}$ School of Business, Belhaven University, USA
}

\begin{abstract}
Many countries were not prepared for a pandemic on June 11, 2009, when the World Health Organization (WHO) declared an Influenza Pandemic. Although Pandemic Influenza Preparedness Planning activity for 2011 has declined compared to 2009, we cannot take preparedness planning off the radar due to future potential pandemics. Unless countries develop model Pandemic Influenza Preparedness Plans, the consequences of being unprepared could be devastating to all of humankind.

This study identified Critical Success Factors (CSFs) necessary at all levels (local, state, and national) to achieve model Pandemic Influenza Preparedness Planning. Pandemic Influenza Preparedness Planning involves developing a plan in the event of the emergence of an influenza virus that causes serious illness and is spread easily and is sustainable among humans. Once the WHO issues a pandemic influenza alert, all countries should be prepared. Data was collected through surveys, interviews, and benchmarking methods. The goal of identifying CSFs is to provide those factors to countries as well as authorities on a local, state, and national level in order to develop model Pandemic Influenza Preparedness Plans.
\end{abstract}

Several CSFs were identified, they included the following: strong leadership support, plan development, having logical response plans, exercising plans, clear operations and implementation policies, adequate budget/resources, effective 
public communications and outreach, and staff training. Clearly, there are benefits to providing CSFs for Pandemic Influenza Preparedness Planning. Being prepared can save the lives of millions around the world and as well as reduce economic and social impact. Pandemic Influenza Preparedness Planning should remain a high priority.

Keywords: influenza, preparedness, biological threats, pandemic, public health.

\section{Introduction}

For many years, there has been considerable international concern regarding the possibility of pandemics occurring in the United States as well as other countries. The World Health Organization (WHO) describes a pandemic using three conditions: the emergence of a disease to the population; the agent infects humans, causing serious illness; and the agent spreads easily and sustainably among humans. The WHO strongly recommends that all countries develop and prepare pandemic plans in advance to prevent and control the next influenza pandemic.

The WHO [1] developed a checklist for influenza pandemic preparedness planning for the benefit of its Member States worldwide. The checklist reflects international expert opinion and includes the following essential elements in the checklist:

- Preparing for an emergency

- Surveillance

- Case investigation and treatment

- Preventing spread of the disease in the community

- Maintaining essential services

- Research and evaluation

- Implementation, testing and revision of the national plan

The capacity of countries for influenza pandemic planning varies, and they may be at different stages of the planning process. The aim of the pandemic preparedness checklist is primarily to provide an outline of the essential minimum elements of preparedness, as well as elements of preparedness that are considered desirable. It is recommended that responsible authorities or institutions in countries that are in the process of planning should consider the specific aspects of the checklist for which they are responsible. Countries that already have a national pandemic preparedness plan in place may use the checklist to evaluate the completeness of the current plan.

The WHO posed the question: Are you prepared? Are you prepared to prevent or minimize the human morbidity and mortality, the social disruption, and the economic consequences caused by an influenza pandemic?

\subsection{Global perspective}

Garrett [2] stated that the world has rapidly become much more vulnerable to the eruption, and most critically, to the widespread and even global spread of both new and old infectious diseases. This new and heightened vulnerability is not 
mysterious. The dramatic increase in worldwide movement of people, goods, and ideas is the driving force behind the globalization of disease. For not only do people travel increasingly, but they travel much more rapidly, and go to many more places than ever before. A person harboring a life-threatening microbe can easily board a jet plane and be on another continent when the symptoms of illness strike. The jet plane itself and its cargo can carry insects bringing infectious agents into new ecologic settings. Few habitats on the globe remain truly isolated or untouched, as tourists and other travelers penetrate into the most remote and previously inaccessible areas in their search for new vistas, business, or recreation.

Such a devastating disease would clearly have profound implications for international relations and the global economy. With death tolls rising, vaccines and drugs in short supply, and the potential for the virus to spread further, governments would feel obliged to take drastic measures that could inhibit travel, limit worldwide trade, and alienate their neighbors. It is even doubtful that any of the world's wealthy nations would be able to meet the needs of their own citizenry, much less those of other countries. Domestic vaccine purchasing and distribution schemes currently primarily assume that only the very young, the elderly, and the immunocompromised are at serious risk of dying from the flu. Every year the United States plans for 185 million vaccine doses, trusting that the flu will kill only the usual risk groups. If that guess were wrong, if all Americans were at risk, the nation would need at least 300 million doses. That is what the entire world typically produces each year. There would thus be a global scramble for vaccine (Garrett [3]).

\subsection{Critical success factors (CSFs)}

There is a need for public and private sectors to take action with pandemic preparedness activities. The identification of Critical Success Factors (CSFs) for pandemic preparedness planning will set a precedent for successful planning of these activities on a local, state, and national level.

CSFs are those few things that must go well to ensure success for a manager or an organization. They represent those managerial or enterprise areas that must be given special and continual attention to bring about high performance. CSFs include issues vital to an organization's current operating activities and to its future success (Friesen and Johnson [4]).

In the case of pandemic preparedness planning, the CSFs would be things and/or tasks that should be identified by organizations to ensure the creation of a successful pandemic preparedness plan. Essentially, highlighting the areas that must be given special and continual attention in order to have a high performing pandemic preparedness plan would constitute the identification of CSFs.

\section{History and current state of Avian Influenza}

Influenza A (H5N1) virus, also called "H5N1 virus", is an influenza A virus subtype that occurs mainly in birds and is highly contagious among birds, and 
can be deadly to them. The H5N1 virus does not usually infect people, but infections with these viruses have occurred in humans. Most of these cases have resulted from people having direct or close contact with $\mathrm{H} 5 \mathrm{~N} 1$ through infected poultry or H5N1 contaminated surfaces. Avian Influenza is also known as "Bird Flu" (Key facts about Avian Influenza (Bird Flu) and Avian Influenza A (H5N1) Virus [5]).

According to Osterholm [6], Avian influenza caused by H5N1 initially received widespread attention in 1997 due to an outbreak in poultry in Hong Kong subsequently spread the virus to humans. There were eighteen human cases recognized; six of the patients died (there was no evidence of person-toperson transmission). In the fall of 2003, H5N1 avian influenza appeared in domestic poultry farms in Asia. It resurfaced in the summer of 2004 after subsiding briefly, appearing in Cambodia, China, Laos, Thailand, and Vietnam, where it persists today despite the prevalent vaccination of poultry. Studies of recent H5N1 isolates in Southeast Asia have indicated that the virus' predominant lineage today originated in southern China. Other lineages are believed to have emerged in Southeast Asia, which suggests that the virus has been present in the region for a long time. A report by the UN Food and Agricultural Organization published in September 2004 found that existing reservoirs of the $\mathrm{H} 5 \mathrm{~N} 1$ influenza virus in ducks, wild birds, and potentially pigs, are already resilient enough to poise a severe challenge to eradication (Osterholm [6]). More recently, Woodward [7] indicated that according to the World Health Organization, as of March 6, 2006, 175 people in seven countries had contracted the H5N1 disease, and 95 people had died. Most of these infections were as a result of people having direct or close contact with H5N1-infected poultry or contaminated surfaces.

Osterholm [6] stated further that, similar to earthquakes, hurricanes, and tsunamis, influenza, pandemics are recurring natural disasters. The natural reservoir of the influenza virus is wild aquatic birds. Therefore, for a human influenza pandemic to occur, a strain of an avian influenza virus must develop to which humans have no pre-existing immunity and undergo critical genetic changes that allow it to be readily transmitted from person to person. The H5N1 strain of the influenza virus has had a limited impact on human health so far, but a human influenza pandemic could occur and be devastating if a current strain underwent the right genetic changes.

For several years, scientists believed that the only way for an avian influenza virus to become transmittable between humans was through a process known as reassortment. Reassortment takes place when an avian virus and a human virus both infect the same cells of an animal or a person and swap genes, producing a new virus adapted to humans. This is how the 1957 and 1968 influenza pandemics began (Osterholm [6]). The Asian Flu of 1957-58 killed 1 million to 2 million worldwide; and the Hong Kong Flu of 1968-69 killed more than 700,000 worldwide (Green [8]). Over the past couple of years, however, the potential development of a human influenza pandemic similar to that of 1918 (due to Spanish influenza) has been a paramount concern. The observation of two of the three key criteria that characterized the pandemic of 1918-1919 has 
already been fulfilled in the present epidemic: (1) the ability of the virus to infect humans resulting in high mortality and (2) a global immunologically naïve human population. The third criterion, efficient human-to-human transmission, has yet to be observed. The adaptation that would result in human-to-human transmission might involve changes in the receptor properties or improved viral replication efficiency. This type of adaptation might be achieved by mutation of an avian virus genome or by mixing segments of an avian virus with segments from a virus already adapted to humans (genetic reassortment), paving the way to the emergence of a new influenza subtype with pandemic potential (Trampuz et al. [9]). According to (Garrett [3]) the 1918-19 Spanish flu killed 50 million people in 18 months; however, Avian Flu is far more dangerous. It kills 100 percent of the domesticated chickens it infects and among humans the disease is also lethal.

Although it is impossible to know for certain whether H5N1 will ever evolve into the next human pandemic virus, more and more of the genetic changes documented in the 1918-1919 Spanish flu have also been found to have occurred in recent $\mathrm{H} 5 \mathrm{~N} 1$ strains affecting both birds and people. Meanwhile, the spread of $\mathrm{H} 5 \mathrm{~N} 1$ infections to more avian species and to more humans continues to point to $\mathrm{H} 5 \mathrm{~N} 1$ as a likely strain of the next pandemic (Osterholm [6]).

\section{History and current state of pandemic influenza preparedness planning}

According to the World Health Organization [10], in the previous years, new strains of influenza have generated pandemics causing extensive death rates and paramount social disruption. In the $20^{\text {th }}$ century, the greatest influenza pandemic occurred in 1918-1919 and caused an estimated 40-50 million deaths all over the world. Although health care has seen some significant improvements in the last decades, epidemiological models from the Centers for Disease Control and Prevention (CDC), Atlanta, USA project that today a pandemic is likely to result in 2 to 7.4 million deaths globally. In high income countries alone, which account for $15 \%$ of the world's population, models project a demand for 134 233 million outpatient visits and 1.5-5.2 million hospital admissions. However, the impact of the next pandemic is likely to be the greatest in low income countries because of different population characteristics and the already strained health care resources.

If an influenza pandemic occurs, the following can be anticipated:

- Given the high level of global traffic, the pandemic virus may spread rapidly, leaving little or no time to prepare.

- Vaccines, antiviral agents and antibiotics to treat secondary infections will be in short supply and will be unequally distributed. It will take several months before any vaccine becomes available.

- Medical facilities will be overwhelmed.

- Widespread illness may result in sudden and potentially significant shortages of personnel to provide essential community services. 
- The effect of influenza on individual communities will be relatively prolonged when compared to other natural disasters, as it expected that outbreaks will reoccur [10].

The World Health Organization (WHO) indicated further that continuous global surveillance of influenza is critical. The WHO has a network of 112 National Influenza Centers that monitors influenza activity and isolates influenza viruses in all continents. The National Influenza Centers will report the emergence of an "unusual" influenza virus immediately to the WHO Global Influenza Programme (a network comprised of 4 WHO Collaborating Centers, WHO CCs, and 122 institutions in 94 countries), or to 1 of the 4 WHO Collaborating Centers (located in Australia, Japan, England and the United States). Rapid detection of unusual influenza outbreaks, isolation of possible pandemic viruses and immediate alert to the WHO system by national authorities is paramount to a timely and efficient response to pandemics (The WHO Global Influenza Surveillance Network [10]).

(Osterholm [6]) indicated that slacking off on preparedness activities today could lead to very bad news in the future. The warning that another pandemic could occur at any time and at a staggering cost to human health and the world economy was direct. These facts remain incontrovertible as many public health scientists believed that the outbreaks of the H5N1 influenza virus in birds in Asia, Europe, and Africa, with occasional infections in humans, were precursors to the next pandemic. Experts have recently noted that it would take only one to six months from the time a human transmitted form of the virus is detected in the United States (U.S.) for it to spread across the country, leaving a short window for hospitals to make preparations. The U.S. government health care agencies have informed businesses to be prepared for a pandemic that might spread to as much as 30 percent to 40 percent of the work force (Green [8]).

\section{Global pandemic influenza preparedness planning}

Mercer [11] invited organizations globally to participate in the Mercer Avian Flu Pandemic Preparedness Survey online. The online survey represented a critical first step in conducting an inventory of the organization's current level of preparedness for a pandemic crisis from a Human Resources (HR) perspective. The findings consisted of data gathered from 450 respondents across 38 countries and 26 industries with respondents from Australia, Canada, People's Republic of China, Hong Kong, Singapore, United Kingdom and the United States comprising 75 percent of total respondents. The top six industries, comprising 60 percent of participants, were manufacturing, finance, professional services, computer services, insurance, and education.

There were five key indicators that Mercer [11] utilized to gauge organizational pandemic preparedness. When observed individually, each of the indicators provided insight into the priorities that organizations have established in their pandemic preparedness planning. When grouped, they provide a broader review of the overall state of an organization's preparedness for an Influenza Pandemic. The key indicators were: 
- Establishment of a budget for pandemic preparedness

- Formation of a crisis leadership management team

- Development of a pandemic business continuity plan

- Workforce planning (including skills inventory)

- Development of an employee communication strategy

There were 11 questions that covered a comprehensive range of topics which included:

- Impact on organizational success factors

- Crisis leadership

- Employee communication

- Skills inventory for workforce planning

- Remote working procedures

- International assignees and corporate travelers

- Compensation and leave policies

- Insurance coverage

- Hygiene, quarantine and preventive health measures

- Employee assistance

- Business continuity planning

The survey report was formulated by identifying themes and issues that evolved from the responses and provided insight into the current state of organizational preparedness for a pandemic. Mercer [11] also believed that the report would provide companies with the ability to benchmark their capability to respond to a pandemic crisis and formulate necessary business continuity planning strategies.

The critical themes that emerged from the report are as follows:

- There is a considerable gap between organizational concern about the impact of a pandemic and organizations' current state of pandemic preparedness.

- Those countries that endured the Asian SARS crises of 2003 are generally more advanced in the pandemic preparedness planning.

- Conversely, for the United States and other economies that were not impacted by SARS and have not had direct exposure to the Avian Flu, planning is in its relative infancy.

- Labor intensive industries have recognized the profound consequences that a pandemic may inflict.

- Although results differ considerably by region and industry type, organizations globally are predicting that a pandemic will result in financial hardship.

\section{Progress report 2008: H5N1/bird flu}

According to the Responses to Avian Influenza and State of Pandemic Readiness, Fourth Global Progress Report [12], a global analysis of the current state of the H5N1 Virus indicates that as recent as mid to late 2008, there have been fewer of the following: outbreaks in poultry, newly infected countries, 
human cases, and deaths compared to the same in 2006 and 2007. Over 50 of the 61 countries that have experienced an H5N1 Virus outbreak have successfully eliminated the disease. However, the H5N1 Virus remains deeply rooted in several countries and the threat of further outbreaks of the virus in poultry (and random cases in humans) persists. This is a confirmation that the threat of an H5N1 (Avian/Bird Flu) influenza pandemic remains imminent.

\subsection{Progress report 2009: H1N1/swine flu}

While the H5N1 Virus remains somewhat dormant, the H1N1 Virus has become more prevalent since April 2009. According to the World Health Organization [13], as of April 26, 2009, the United States Government had reported 20 laboratory confirmed human cases of swine influenza A/H1N1 at that time (8 in New York, 7 in California, 2 in Texas, 2 in Kansas and 1 in Ohio). All 20 cases had mild Influenza-Like Illness with only one requiring brief hospitalization and no deaths reported. All viruses had the same genetic pattern based on preliminary testing. The virus was being described as a new subtype of A/H1N1 not previously detected in swine or humans. The WHO reported further that on April 26, 2009, the Government in Mexico had reported 18 laboratory confirmed cases of swine influenza $\mathrm{A} / \mathrm{H} 1 \mathrm{~N} 1$. Investigation was continuing to clarify the spread and severity of the disease in Mexico. There were suspect clinical cases reported in 19 of the country's 32 states at that time.

The World Health Organization (WHO) declared an influenza pandemic on June 11, 2009. The level of influenza pandemic alert was raised from phase 5 to phase 6 (pandemic period). At that time, nearly 30,000 confirmed cases had been reported in 74 countries. This particular H1N1 strain was entirely new and had not circulated previously in humans. It was indicated further that it was contagious and easily spreading from one person to another (WHO [13]).

The World Health Organization (WHO) informed that countries should prepare to see cases, or the further spread of cases, in the near future. Countries where outbreaks appear to have peaked should prepare for the second wave of infection. The WHO stated further that guidance on specific protective and precautionary measures had been sent to ministries of health in all countries (WHO [13]).

\subsection{Progress report 2010: H1N1/swine flu}

According to the World Health Organization [14], more than 209 countries and overseas territories have reported laboratory confirmed cases of the H1N1 2009 flu; this includes 14,142 deaths worldwide as of January 22, 2010. At that time, the most concentrated transmission of the virus continued to occur in North America, South Asia, and in limited areas of Eastern Europe. Overall, pandemic influenza activity in the temperate northern hemisphere reached a peak between late October and late November and has continually declined since. However, the Global Influenza Surveillance Network (GISN) continues global circulation of influenza viruses. 
More recently, as of May 21, 2010, the World Health Organization continues to report deaths from laboratory confirmed cases of the 2009 H1N1 flu. These deaths are an under-representation of true numbers in that many deaths are not tested or confirmed as influenza related. The more active areas of transmission are in the tropical regions of the Caribbean, South America, and Southeast Asia (CDC [15]).

According to the Washington Post [16], the Obama Administration's fast and effective response to the $2009 \mathrm{H} 1 \mathrm{~N} 1$ flu outbreak last year was the payoff of preparedness efforts since 2005 . The government issued a strategy to prepare for a potential pandemic flu in 2005 and there have been significant improvements in the following areas: surveillance, coordination, communications, treatment capabilities and stockpiles, vaccine manufacturing capacity, and ensuring that every state now has a pandemic plan. However, the H1N1 flu has not truly tested the limits of the response plan in that the potential of what could be quite devastating has not taken place.

The Washington Post (2009) stated further that a number of key areas still need improvement including full funding for research to produce a vaccine against a novel virus, building stockpiles of anti-viral medication, so that every state is equally protected, supplying enough annual resources to support ongoing state and local response capacity, managing mass hospitalizations if there is a surge in patients, and the public health workforce during this time of downsizing and layoffs. There are challenges yet to be addressed in anticipation of the next influenza pandemic.

\section{Data analysis and results}

The following research question was addressed in this study: What are the Critical Success Factors (CSFs) in Pandemic Influenza Preparedness Planning? The first research objective of this study was to identify CSFs that are necessary to enhance and implement a Pandemic Influenza Preparedness Plan. The second objective was to increase the awareness and the current knowledge of Pandemic Influenza Preparedness Planning. The final objective of this study was to further necessary research on Pandemic Influenza Preparedness Planning.

The research question for this study was answered by the use of a multimethod approach which utilized the concept of triangulation. The triangulation concept involved combining surveys, interviews, and benchmarking data in order to create meaningful information. Existing information was gathered from current model pandemic influenza preparedness plans and was analyzed and related to primary data collected from this study where appropriate.

Five subject matter experts (panel of experts) from organizations that are responsible for pandemic influenza preparedness planning provided their top five Critical Success Factors (CSFs) and definitions for each CSF they indicated. The participants' open-ended responses were analyzed for content. All of the participants' CSF definitions were compiled and analyzed in random order so that the participant's affiliation would not bias the researcher. First, open coding was performed as a first attempt to condense participants' open-ended responses 
into conceptual categories. Emerging themes were brought to surface during this process and initial codes were assigned. A second analysis led to a reconceptualization of themes and reassigning of codes. This analysis revealed eight different reoccurring themes among the CSFs that were mentioned. These conceptual categories included the following: (1) strong leadership support; (2) plan development; (3) having logical response plans; (4) exercising plans; (5) clear operations and implementation policies; (6) adequate budget/resources;

(7) effective public communications and outreach; and (8) staff training.

\subsection{Summary of key findings}

The present research drew on various sources to determine what factors at the local, state, national (provided by the experts), and international levels are critical to pandemic influenza preparedness planning. The information obtained from literature reviews (model plans), expert panelists, and the benchmark organization (World Health Organization) suggests that there seems to be some agreement across these various organizations in terms of what are those factors. As shown in Table 1, all agree that having clear operations and implementation policies, staff training, plan development, effective public communication and outreach, and logical response plans are important CSFs for pandemic influenza preparedness planning.

Table 1: Critical success factors common across local, state, national, and international organizations.

\begin{tabular}{|c|c|c|c|}
\hline Critical Success Factors & Experts & $\begin{array}{c}\text { State and } \\
\text { Local Model } \\
\text { Organizations }\end{array}$ & $\begin{array}{l}\text { Benchmark } \\
\text { Organization } \\
\text { World Health } \\
\text { Organization }\end{array}$ \\
\hline $\begin{array}{l}\text { Clear Operations and Implementation } \\
\text { Policies }\end{array}$ & $\checkmark$ & $\checkmark$ & $\checkmark$ \\
\hline $\begin{array}{l}\text { Effective Public Communications and } \\
\text { Outreach }\end{array}$ & $\checkmark$ & $\checkmark$ & $\checkmark$ \\
\hline Staff Training & $\checkmark$ & $\checkmark$ & $\checkmark$ \\
\hline Plan Development & $\checkmark$ & $\checkmark$ & $\checkmark$ \\
\hline Having Logical Response Plans & $\checkmark$ & $\checkmark$ & $\checkmark$ \\
\hline Adequate Budget/Resources & $\checkmark$ & & $\checkmark$ \\
\hline Exercising Plans & $\checkmark$ & $\checkmark$ & \\
\hline Strong Leadership Support & $\checkmark$ & & \\
\hline
\end{tabular}

\section{Conclusion}

The research question answered by this study was, "What are the critical success factors in pandemic influenza preparedness planning?" The first objective was to identify the CSFs necessary to enhance and implement a pandemic influenza preparedness plan. The second objective was to increase the awareness and the current knowledge of pandemic influenza preparedness planning. The final objective was to further necessary research on pandemic influenza preparedness 
planning. The research question was answered and the three research objectives were achieved for this study.

While the study answered questions, it raised many questions as well. Questions have been raised still for some, regarding the lack of planning or progress of influenza preparedness planning. There is a considerable gap between concern about the impact of a pandemic and the current state of pandemic preparedness. How can lessons be learned from model pandemic influenza preparedness planning and applied to invoke the urgency in planning on all levels (local, state, and national)?

The consequences of being unprepared for an influenza pandemic can be devastating to all of human-kind. The timeliness of this study is critical with the recent influenza pandemic alert that occurred in June 2009. As we approach another influenza season in 2011, all levels (local, state, and national) of planning should be considered an urgent priority in preparedness of the next potential influenza pandemic. With the identification of the CSFs revealed in this study, preparedness planning on all levels can be fully developed or further enhanced for successful pandemic influenza preparedness planning.

\section{References}

[1] WHO checklist for influenza pandemic preparedness planning. (2005). Retrieved July 28, 2008, from http://www.who.int/csr/resources/ publications/influenza/WHOCDS_CSR_GIP_2005_4/en/

[2] Garret, L. (1994). The coming plague. New York, New York: Penguin Books.

[3] Garrett, L. (2005). The next pandemic? Foreign Affairs 84,(4), 3-23.

[4] Friesen, M. E. and Johnson, J. A. (1995). The success paradigm. Westport, Connecticut: Quorum Books.

[5] Key facts about avian influenza (bird flu) and avian influenza A (H5N1) Virus. (2007). Retrieved March 18, 2008, from http://www.cdc.gov/flu/ avian/gen-info/facts.htm

[6] Osterholm, M. (2007). Unprepared for a pandemic. Foreign Affairs 86, (2), 47-57.

[7] Woodward, N. H. (2006). Pandemic. HR Magazine. 51, (5), 46-52.

[8] Green, E. (2006). Pandemic flu preparations taking shape at area hospitals. Business First (June).

[9] Trampuz, A., Prabhu, R., Smith, T., and Baddour, L. (2004). Avian influenza: a new pandemic threat? Mayo Foundation for Medical Education and Research. 79, 523-530.

[10] World Health Organization. (2008). The WHO global influenza surveillance network. Retrieved July 28, 2008, from http://www.who.int/csr/disease/influenza/influenzanetwork/en/

[11] Mercer. (2006). Avian Flu Pandemic Preparedness Survey Report. New York, NY.

[12] UN System Influenza Coordinator and the World Bank. (2008). Responses to Avian Influenza and State of Pandemic Readiness Fourth Global 
102 Environmental Health and Biomedicine

Progress Report. Retrieved February 4, 2009, from http://siteresources.worldbank.org/EXTAVIANFLU/Resources/31244401172616490974/Fourth_progress_report_second_printing.pdf

[13] WHO Swine flu illness in the United States and Mexico - update 2. (2009). Retrieved April 27, 2009, from http:/www.who.int/csr/don /2009_04 26/en/index.html

[14] WHO Pandemic (H1N1) 2009 - update 84. (2010). Retrieved May 24, 2010, from http://www.who.int/csr/don/2010_01_22/en/index.html

[15] Centers for Disease Control and Prevention 2009 H1N1 Flu: International Situation Update. (2010). Retrieved May 24, 2010, from http://www.cdc.gov/h1n1flu/updates/international/.

[16] Levi, J. (2009). Is America Prepared for a Pandemic. The Washington Post. (April). 